九州大学学術情報リポジトリ

Kyushu University Institutional Repository

\title{
Particle simulation of dual-scale flow in resin transfer molding for process analysis
}

Yashiro, Shigeki

Department of Aeronautics and Astronautics, Kyushu University

Nakashima, Daichi

Graduate School of Integrated Science and Technology, Shizuoka University

Oya, Yutaka

Department of Aerospace Engineering, Tohoku University

Okabe, Tomonaga

Department of Aerospace Engineering, Tohoku University

他

http://hdl. hand le. net/2324/4476067

出版情報: Composites Part A: Applied Science and Manufacturing. 121，pp.283-288，2019-06-01. Elsevier

バージョン :

権利関係 : 


\section{Accepted Manuscript}

Particle simulation of dual-scale flow in resin transfer molding for process analysis

Shigeki Yashiro, Daichi Nakashima, Yutaka Oya, Tomonaga Okabe, Ryosuke Matsuzaki

PII: S1359-835X(19)30118-6

DOI: https://doi.org/10.1016/j.compositesa.2019.03.038

Reference: JCOMA 5392

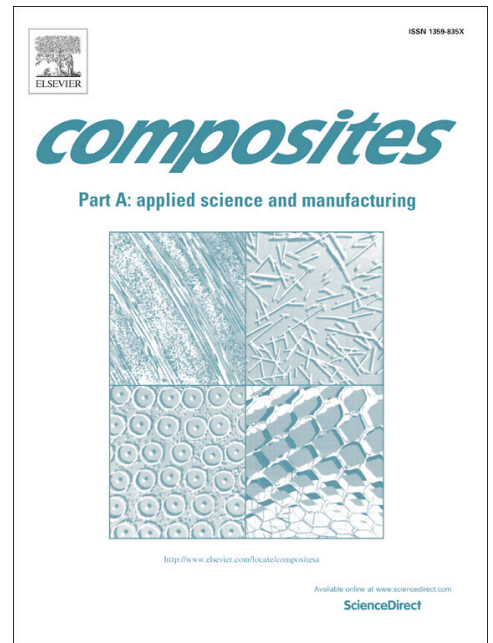

To appear in:

Composites: Part A

Received Date:

12 December 2018

Revised Date:

21 February 2019

Accepted Date:

25 March 2019

Please cite this article as: Yashiro, S., Nakashima, D., Oya, Y., Okabe, T., Matsuzaki, R., Particle simulation of dual-scale flow in resin transfer molding for process analysis, Composites: Part A (2019), doi: https://doi.org/ 10.1016/j.compositesa.2019.03.038

This is a PDF file of an unedited manuscript that has been accepted for publication. As a service to our customers we are providing this early version of the manuscript. The manuscript will undergo copyediting, typesetting, and review of the resulting proof before it is published in its final form. Please note that during the production process errors may be discovered which could affect the content, and all legal disclaimers that apply to the journal pertain. 


\section{Particle simulation of dual-scale flow in resin transfer molding for process analysis}

Shigeki Yashiro ${ }^{1, *}$, Daichi Nakashima ${ }^{2}$, Yutaka Oya ${ }^{3}$, Tomonaga Okabe ${ }^{3}$, Ryosuke Matsuzaki ${ }^{4,5}$

${ }^{1}$ Department of Aeronautics and Astronautics, Kyushu University 744 Motooka, Nishi-ku, Fukuoka 819-0395, Japan

${ }^{2}$ Graduate School of Integrated Science and Technology, Shizuoka University

3-5-1 Johoku, Naka-ku, Hamamatsu 432-8561, Japan

${ }^{3}$ Department of Aerospace Engineering, Tohoku University

6-6-01 Aoba-yama, Aoba-ku, Sendai 980-8579, Japan

${ }^{4}$ Department of Mechanical Engineering, Tokyo University of Science

Yamazaki 2641, Noda, Chiba 278-8510, Japan

${ }^{5}$ Institute of Fluid Science, Tohoku University

2-2-1 Katahira, Aoba-ku, Sendai 980-8577, Japan

* Corresponding author: yashiro@aero.kyushu-u.ac.jp (S. Yashiro)

\section{Abstract}

Modeling the inhomogeneous microstructures of fibrous tows is important for analyzing the process of resin transfer molding because dual-scale pores in a preform can lead to void formation. This study focused on the development of a microscopic flow analysis method to predict the impregnation of fiber bundles. The moving particle semi-implicit method was adopted to model the microstructure of a fiber bundle explicitly and inter-particle potential 
force was introduced into the numerical model to take account for the capillary effect. The predicted process of impregnation and void formation agreed with empirical observations. The developed approach was applied to predict the relationship between the modified capillary number and void content to identify the optimal molding conditions to reduce microvoids. The obtained relationship reproduced the trends of a reported experiment, which indicates that the proposed approach will provide information about optimal conditions for minimizing void content.

Keywords: A. Polymer-matrix composites (PMCs); B. Wettability; C. Process simulation; E. Resin flow

\section{Introduction}

Because fiber-reinforced plastics are lightweight and have desirable mechanical properties, they have been widely adopted in aerospace and automobile structures to improve fuel efficiency. More cost-effective molding processes compared to prepreg-based autoclave molding are required for the mass production of automobiles and next-generation aircraft. Resin transfer molding (RTM) is a promising method based on its high productivity and the excellent mechanical properties of manufactured composites. However, voids may be formed in or near fiber tows during the impregnation process, which deteriorates the mechanical 
properties of the composites [1]. The formation of such defects is an obstacle to the widespread use of liquid molding processes.

The voids formed during RTM are classified depending on their scale. Macrovoids (also called dry spots) are large air pockets across fiber bundles, which are caused by incomplete resin filling. Mesovoids and microvoids are formed based on molding conditions and the microstructure of the fibrous preform. Dual-scale pores in the preform lead to different permeability values inside and between fiber tows, which leads to air entrapment during the resin filling process. Mesovoids are air bubbles between fiber bundles that are generated when the flow rate inside the fiber bundles is higher than that outside the bundles. Microvoids are tiny air residuals inside fiber bundles that are formed when the flow rate outside the fiber bundles is higher than that inside the bundles. The balance of viscous flow and capillary flow governs void formation and is important for the reduction of void content.

There have been numerous studies focusing on the experiment, analytical modeling and numerical simulation of void formation and the optimization of the RTM process [2]. Sadiq et al. [3] experimentally determined that low local permeability in a dense bundle causes void formation in fiber bundles. Patel and Rohatgi [4,5] observed the impregnation of unidirectional stitched fiberglass mats and determined that the fraction of mesovoids and microvoids is correlated with the modified capillary number, which is the ratio of viscous 
force to capillary force. Microscopy analysis of a chopped-strand randomly oriented mat revealed significant void variation in the through-thickness (impregnation) direction [6]. In-situ observations of the filling process of woven fabrics have revealed that mesovoids are formed by the dominant capillary flow and lead-lag flow front [7]. Mathematical models for analyzing void formation have been developed by considering microscale flow into inhomogeneous preforms based on Darcy's law. Kang et al. [8] predicted the relationship between the void fraction and modified capillary number by analyzing time differences when filling a bundle and open channel. Matsuzaki et al. $[9,10]$ developed a similar mathematical model using a mathematical permeability model [11] and their prediction of the fraction of mesovoids based on flow velocity agreed with experimental measurements. Lebel et al. [12] applied a mechanistic imbibition model to fabrics to characterize the impregnation process and determined that two key parameters, namely the modified capillary number and penetrativity of the fluid, should be optimized to reduce voids. They also proposed an experimental method to obtain the optimal conditions for a given resin-fabric pair.

RTM simulations based on the finite-element/control-volume (FE/CV) method [13-15] have been developed over the past few decades to predict void content. In the standard approach, the governing equation for single-phase flow is solved on the macroscopic scale, which essentially neglects microscale pores in the bundle. This approach has been extended to 
involve the microscale intra-tow flow and to predict microvoids and mesovoids [16-19].

Although the optimal value of the modified capillary number has been predicted using FE/CV methods [18], such methods always require a measured value of local permeability or model of permeability as input data. Proper calibration may be required to apply these permeability models $[11,20,21]$ to a real preform.

To overcome this difficulty, this study presents an impregnation analysis method from a microscopic viewpoint. In recent years, microscopic flow simulation in a fiber array has been investigated by solving the momentum equation using the finite-element method and finite-volume method, and the effective permeability have been analyzed based on the intra-tow flow [22-26]. A particle method is employed in this study to explicitly model the microstructures of fibrous tows and analyze the void formation process based on the flow through open channels and intra-tow paths. The surface tension effect can be easily introduced into particle simulations, and this characteristic enables us to predict the entrapment of air without using permeability. Okabe et al. [27] proposed a method for analyzing microscopic flows through fibrous tow based on the moving particle semi-implicit (MPS) method [28] and considered the capillary effect as a potential force. However, the results did not reproduce the mechanisms of air entrapment during RTM [4,5] accurately because the open channels between bundles were neglected. Lu et al. [29] presented a flow analysis of the RTM process 
using smoothed particle hydrodynamics, but they neglected the capillary effect, which is essential to estimating optimal RTM process conditions.

Therefore, this study focused on the development of a numerical method for analyzing microscale flows during the RTM process. To this end, we adopted the MPS method to explicitly model the microstructures of fiber bundles and incorporated inter-particle potential force into the MPS method to account for the capillary effect. This remainder of this paper is organized as follows. Section 2 describes experiments on liquid flow using a transparent mold with simulated fiber bundles. Section 3 briefly introduces the MPS method and an inter-particle potential force related to surface tension and wettability. In Section 4, flow analysis corresponding to the experiments is performed to examine the process of microvoid formation. A numerical study is presented in Section 5, where we estimate optimal molding conditions to reduce the void fraction without using permeability.

\section{Experiment}

To observe the process of void formation in a fiber bundle, a transparent polymethyl methacrylate mold with simulated fiber bundles (Fig. 1) was prepared. The mold cavity space was $22.0 \mathrm{~mm}$ wide and $1.0 \mathrm{~mm}$ thick. Quartz rods with a diameter of $1.0 \mathrm{~mm}$ were embedded into the mold and the minimum spacing between adjacent rods was $0.4 \mathrm{~mm}$. The space 
between adjacent bundles (blocks with densely arranged rods) was $2.5 \mathrm{~mm}$ in the width direction. The local volume fraction of the rods in each bundle was $50 \%$ and the nominal rod volume fraction in the rods arranged area was $27 \%$.

Liquid was injected from one end of the mold (left side in Fig. 1a) and the impregnation process was observed. In this study, water and olive oil were used as test liquids, which were chosen by considering their different viscosities and resultant capillary effects. Their properties are listed in Table 1 . The liquids were injected by a micro-syringe pump at a constant flow rate of $500 \mathrm{ml} / \mathrm{h}$. This flow rate corresponds to a flow-front velocity of 6.3 $\mathrm{mm} / \mathrm{s}$ in the empty space. A high-speed digital microscope (VW-9000, Keyence) was used to observe the flow. The impregnation of the bundle shown in Fig. 1a was observed in detail and the void fraction was determined as the area of the voids divided by the observed rectangle area, in which the nominal rod volume fraction was $30 \%$.

Figures 2 presents snapshots of the observed water flow into the bundle, where the dotted line is the flow front of the liquid. Water impregnated only the outermost layer of bundles and then flowed between bundles without further impregnation. The flow velocity in the open channels was higher than that in the bundles and the bundles were surrounded by water before complete saturation occurred. As a result, air remained inside the bundles and the void fraction of the observed area was $10.8 \%$. This mechanism of microvoid formation agrees with 
previous experiments [3,5]. Figure 3 presents snapshots of the oil injection experiment. In contrast to the results of water injection, the oil flowed into the bundles. Although the flow front in the open channels still moved faster than that inside the bundles, the velocity difference was small. Therefore, the bundles were filled completely, and no voids were formed. The capillary effect is the main cause for this difference between the two liquids [30].

\section{Analysis}

Microscale flow in fibrous media as well as flow of fiber suspension have been studied using particle simulation methods owing to their capability to represent microstructures [31]. In particular, the lattice Boltzmann method [32], the smoothed particle hydrodynamics [29] and the MPS method [27] have been used for those analyses. Among them, this study employs the MPS method for the analysis of inter- and intra-tow flow, because it is suitable for solving incompressible flow and easy to introduce the capillary force into the analysis

framework.

\subsection{MPS method}

The MPS method [28] is a particle-based method in which a continuum is expressed as an assembly of particles. Each particle is assumed to have an effective radius for particle 
interaction and differential models are prepared based on particles within the effective volume.

When a particle $i$ has a scalar variable $\phi_{i}$ and a vector variable $\mathbf{u}_{i}$, the corresponding differential models are defined as follows:

$$
\begin{gathered}
\langle\nabla \phi\rangle_{i}=\frac{d}{n^{0}} \sum_{j \neq i}^{N}\left[\frac{\phi_{j}-\phi_{i}}{\left|\mathbf{r}_{j}-\mathbf{r}_{i}\right|^{2}}\left(\mathbf{r}_{j}-\mathbf{r}_{i}\right) w\left(\left|\mathbf{r}_{j}-\mathbf{r}_{i}\right|\right)\right] \\
\langle\nabla \cdot \mathbf{u}\rangle_{i}=\frac{d}{n^{0}} \sum_{j \neq i}^{N}\left[\frac{\left(\mathbf{u}_{j}-\mathbf{u}_{i}\right) \cdot\left(\mathbf{r}_{j}-\mathbf{r}_{i}\right)}{\left|\mathbf{r}_{j}-\mathbf{r}_{i}\right|^{2}} w\left(\left|\mathbf{r}_{j}-\mathbf{r}_{i}\right|\right)\right] \\
\left\langle\nabla^{2} \phi\right\rangle_{i}=\frac{2 d}{\lambda n^{0}} \sum_{j \neq i}^{N}\left[\left(\phi_{j}-\phi_{i}\right) w\left(\left|\mathbf{r}_{j}-\mathbf{r}_{i}\right|\right)\right] \\
\lambda=\int_{r_{0}}^{r_{e}} r^{2}\left(\frac{r_{e}}{r}-1\right) 2 \pi r \mathrm{~d} r / \int_{r_{0}}^{r_{e}}\left(\frac{r_{e}}{r}-1\right) 2 \pi r \mathrm{~d} r
\end{gathered}
$$

Here, $d$ is the dimensional number, $n_{0}$ is the constant particle number density under incompressible conditions, $N$ is the number of particles within the effective radius $r_{e}, \mathbf{r}$ is the position vector, and $w\left(=r_{e} / r-1\right)$ is the weight function.

The governing equations for incompressible flow are the mass conservation and the

\section{Navier-Stokes equation:}

$$
\begin{gathered}
\frac{D \rho}{D t}=0, \\
\frac{D \mathbf{u}}{D t}=-\frac{1}{\rho} \nabla P+v \nabla^{2} \mathbf{u}+\mathbf{g},
\end{gathered}
$$

where $t$ is time, $\rho$ is density, $\mathbf{u}$ is velocity, $P$ is pressure, $v$ is kinematic viscosity, and $\mathbf{g}$ is acceleration caused by an external force, such as gravity. These values are discretized by differential operator models. In the standard MPS method, the viscous (second) and external 
force (third) terms on the right of Eq. (6) are solved explicitly and the Poisson's equation for pressure derived from the pressure (first) term and Eq. (5) is solved implicitly. However, analyzed pressure distributions frequently include numerical oscillations. This study employed an improved solving scheme [33] to obtain a smoother pressure distribution.

\subsection{Inter-particle potential force}

To analyze the capillary effect, an inter-particle potential force was introduced into the MPS method. The inter-particle potential force $\mathbf{F}_{s}$ is defined as the gradient of the potential $\Pi$.

This study employed the Morse potential, which is an interatomic interaction model.

$$
\begin{gathered}
\mathbf{F}_{s}=\sum_{j \neq i}^{N} \frac{\partial \Pi}{\partial\left(\left|\mathbf{r}_{j}-\mathbf{r}_{i}\right|\right)} \cdot \frac{\mathbf{r}_{j}-\mathbf{r}_{i}}{\left|\mathbf{r}_{j}-\mathbf{r}_{i}\right|} \\
\Pi=C\left\{e^{-2\left(r / l_{0}-1\right)}-2 e^{-\left(r / l_{0}-1\right)}\right\},
\end{gathered}
$$

where $C$ is the coefficient, $r$ is the distance between two particles, and $l_{0}$ is the equilibrium distance (i.e., initial particle spacing). The Morse potential, Eq. (8), was adopted with consideration for the stability of analysis.

Wettability is calculated using the same potential model. Wettability can be represented by using two specific potential coefficients for the interaction between two fluid particles and for the interaction between a fluid particle and solid particle, respectively. This study used the following relationship between the two coefficients: 


$$
C_{f s}=\alpha C_{f f},
$$

where $C_{f f}$ and $C_{f s}$ are the coefficients for fluid-fluid interaction (i.e., surface tension) and fluid-solid interaction, respectively. $C_{f f}$ is only used in Eq. (8) if the particle of interest $i$ and its neighboring particle $j$ are both fluid particles. $C_{f s}$ is used when either $i$ or $j$ are solid (Fig. 4). The arbitrary positive constant $\alpha$ was calibrated based on droplet collapse simulations to obtain an appropriate contact angle. The inter-particle potential force was adopted as the external force term in the Navier-Stokes equation.

The potential model was verified through droplet collapse. The contact angles between the liquids and a quartz plate were measured by an automatic contact angle meter (DMe-201, Kyowa Interface Science) and found to be $39.9^{\circ}$ and $27.4^{\circ}$ for water and olive oil, respectively. The collapse of a droplet with a diameter of $2 \mathrm{~mm}$ was then analyzed using $0.125-\mathrm{mm}$ particles. The contact angles for analysis were determined using the half-angle method and were averaged during the period of $0.2-0.5 \mathrm{~s}$, during which the motion of the droplet was mostly converged. Figure 5 depicts the observed droplet shape and the predicted result with adjusted parameters. The predicted contact angles $\left(38.3^{\circ}\right.$ and $30.7^{\circ}$ for water and oil) were matched to the measured angles by adjusting the parameters $C_{f f}$ and $\alpha$ (Table 2). However, the potential model needs to be improved in order to reproduce the observed droplet shape. 


\section{Liquid flow in a fiber bundle}

Two-dimensional analysis of liquid flow into a fiber bundle was performed to predict the void formation process. Figure 6 depicts the analytical model that corresponds to this experiment. The particle size was $0.125 \mathrm{~mm}$ and a single rod was represented by 52 particles. The total number of particles in the initial condition was 8364 . Liquid particles were injected with a velocity of $6.3 \mathrm{~mm} / \mathrm{s}$ from the left side of the model. Specifically, the left wall moved in the injection direction at the prescribed velocity. When the travel distance reached the size of a particle, the wall returned its original position and new fluid particles were generated in the empty space. A periodic boundary condition was applied to the top and bottom ends of the model. An outlet boundary condition was imposed on the right end of the model, and particles passing through the boundary were excluded from the analysis. Water and olive oil were used as test liquids again with the properties listed in Table 1. The inter-particle potential model was considered and the parameters listed in Table 2 were used. For the sake of simplicity and stability of analysis, the density of air was set to the same value as that of water and its kinetic viscosity was set to $1.515 \times 10^{-5} \mathrm{~m}^{2} / \mathrm{s}$. The inter-particle force was not applied to the air particles. The void fraction was calculated as the number of air particles divided by the initial number of particles in the designated region.

Figure 7 depicts snapshots of the predicted water flow into the bundle. When the water 
reached the left end of the bundle, it impregnated up to one layer inside the outermost layer of the bundle (Fig. 7a). The bundle was hardly immersed in the injection direction and water flowed mainly through the open channels, as depicted in Figs. $7 \mathrm{~b}$ and $7 \mathrm{c}$. The bundle was finally surrounded by water and air remained inside the bundle (Fig. 7d). This process of microvoid formation agrees well with the experimental results (Fig. 2). The predicted void fraction in the final state was $9.4 \%$, which also agrees with the experimental results.

Figure 8 presents snapshots of the predicted flow of oil into the bundle. Similar to the experimental results, impregnation proceeded from the left end of the bundle until $0.6 \mathrm{~s}$ (Figs. $8 \mathrm{a}$ and $8 \mathrm{~b})$. The bundle was then immersed in the direction perpendicular to injection because of the capillary effect and a small amount of air was entrapped between the fibers (Fig. 8c). The flow front velocity in the open channels was slightly higher than the impregnation speed and the bundle was surrounded by the oil before all air was ejected (Fig. 8d). The amount of entrapped air differed from the experimental results, but most of the bundle was filled and the void fraction was $2.9 \%$.

Although there was a small error in the void fraction, the overall impregnation process agreed with the experimental results in Section 2. Therefore, flow analysis with an adequate capillary effect model can predict change in the fraction of microvoids in fiber bundles under varying molding conditions. Dimensionless analysis to address the difference in scale from an 
actual fiber bundle and verification of the simplified air viscosity are our future work.

\section{Discussion}

Although the proposed approach reproduced the process of microvoid formation successfully, it can analyze only a small portion of a real mold based on its high calculation cost. The FE/CV method [13-15] is realistic for predicting the molding process of a structural member from the viewpoint of the calculation cost, and the present approach can be used as a convenient auxiliary tool. The FE/CV method requires the permeability of a fiber preform as input data, which is typically measured experimentally. Furthermore, optimal molding conditions can be specified after a number of molding experiments and observations for each combination of fiber preform and resin. Microscale flow analysis can be used as a substitute for such laborious experiments. The remainder of this section will demonstrate such an application of the proposed approach.

The process of air entrapment varies based on molding conditions. The void fraction in an RTM process can be determined based on the modified capillary number $\mathrm{Ca}^{*}$ as follows:

$$
C a^{*}=\frac{\mu u}{\sigma \cos \theta}
$$

where $\mu$ is the viscosity, $u$ is the superficial velocity (injection flow velocity in this study), $\sigma$ is the surface tension, and $\theta$ is the contact angle. 
A numerical study was performed to predict the optimal conditions from the perspective of microvoid formation. Figure 9 depicts the analytical model. The size of each particle was 2 $\mu \mathrm{m}$ and seven particles were arranged in a hexagonal shape to approximate a fiber with a diameter of $6 \mu \mathrm{m}$. There were 3942 particles in the initial state. Fluid particles were injected from the left end of the model and the periodic boundary condition was applied to the top and bottom of the model. The void fraction in the fiber bundles was predicted under 27 different conditions with varying injection speeds $(1,10$, and $100 \mathrm{~mm} / \mathrm{s})$, viscosities $(0.1,0.5$, and 1 $\mathrm{Pa} \cdot \mathrm{s})$, and contact angles $\left(40^{\circ}, 60^{\circ}\right.$, and $\left.80^{\circ}\right)$. The density and surface tension of the fluid were constant $\left(1000 \mathrm{~kg} / \mathrm{m}^{3}\right.$ and $7.28 \times 10^{-2} \mathrm{~N} / \mathrm{m}$, respectively). These conditions correspond to a modified capillary number range of $1.8 \times 10^{-2}-7.9$. The number of air particles remaining in the region shown in Fig. 9 was counted after the major flow front reached the right end of the model.

Figure 10a presents an example of the final particle distribution; air was entrapped in the bundles under the condition $C a^{*}=7.9$, similar to the observation (Fig. 2). The predicted relationship between the void fraction and modified capillary number is plotted in Fig. 10b. The void fraction was zero at $\mathrm{Ca}^{*}$ values less than 0.01 and increased monotonically in with $\mathrm{Ca}^{*}$ values greater than 0.02 . This trend in the void fraction agrees with reported experimental data on microvoids [5]. Therefore, flow analysis considering the microstructure of the 
preform and capillary effect has possibility of predicting optimal molding conditions to minimize microvoids. However, this analysis could not reproduce the mesovoids observed in past experiments [4]. This difference is the result of the analytical model disregarding the woven structures of bundles. Warp yarns should be included in the model to predict the relationship between the void fraction and $\mathrm{Ca}^{*}$ within a low capillary number range. It should be noted that the optimal $\mathrm{Ca}^{*}$ value will depend on the number of fibers in a tow.

\section{Conclusions}

This study focused on the development of a flow analysis method to predict void formation during the RTM process using the MPS method, which enabled us to explicitly model the microstructures of fiber bundles. An inter-particle potential force was introduced into the MPS method to account for the capillary effect. The impregnation of simulated fiber bundles was observed in a transparent mold to verify the proposed analytical method. Our conclusions are

summarized below.

1. The developed flow analysis method with microstructure models of fiber preforms reproduced the process of microvoid formation in fiber bundles observed in experiments successfully. The predicted impregnation and void formation processes agreed with our experimental observations. 
2. The relationship between the void fraction and modified capillary number was predicted by the developed analysis method successfully. The predicted results reproduced the trends observed during past experiments on microvoids [5]. This demonstrates the ability of the proposed method to predict optimal molding conditions to minimize the void content in RTM processes.

\section{Acknowledgement}

This work was supported by the Cross-ministerial Strategic Innovation Promotion (SIP) Program, "Structural Materials for Innovation."

\section{References}

[1] Leclerc JS, Ruiz E. Porosity reduction using optimized flow velocity in Resin Transfer Molding. Compos Part A 2008; 39: 1859-1868.

[2] Park $\mathrm{CH}$, Lee WI. Modeling void formation and unsaturated flow in liquid composite molding processes: a survey and review. J Reinf Plast Compos 2011; 30: 957-977.

[3] Sadiq TAK, Advani SG, Parnas RS. Experimental investigation of transverse flow through aligned cylinders. Int J Multiphase Flow 1995; 21: 755-774.

[4] Patel N, Rohatgi V, Lee LJ. Micro scale flow behavior and void formation mechanism 
during impregnation through a unidirectional stitched fiberglass mat. Polym Eng Sci 1995; 35: 837-851.

[5] Rohatgi V, Patel N, Lee LJ. Experimental investigation of flow-induced microvoids during impregnation of unidirectional stitched fiberglass mat. Polym Compos 1996; 17: 161-170.

[6] Hamidi YK, Aktas L, Altan MC. Formation of microscopic voids in resin transfer molded composites. J Eng Mater Technol 2004; 126: 420-426.

[7] Matsuzaki R, Seto D, Todoroki A, Mizutani Y. Void formation in geometry-anisotropic woven fabrics in resin transfer molding. Adv Compos Mater 2014; 23: 99-114.

[8] Kang MK, Lee WI, Hahn TH. Formation of microvoids during resin-transfer molding process. Compos Sci Technol 2000; 60: 2427-2434.

[9] Matuzaki R, Seto D, Naito M, Todoroki A, Mizutani Y. Analytical prediction of void formation in geometrically anisotropic woven fabrics during resin transfer molding. Compos Sci Technol 2015; 107: 154-161.

[10]Matsuzaki R, Naito M, Seto D, Todoroki A, Mizutani Y. Analytical prediction of void distribution and a minimum-void angle in anisotropic fabrics for radial injection resin transfer molding. Express Polym Lett 2016; 10: 860-872.

[11]Gebart BR. Permeability of unidirectional reinforcements for RTM. J Compos Matter 
1992; 26: 1100-1133.

[12]LeBel F, Fanaei AE, Ruiz É, Trochu F. Prediction of optimal flow front velocity to minimize void formation in dual scale fibrous reinforcements. Int $\mathbf{J}$ Mater Form 2014; 7: 93-116.

[13]Bruschke MV, Advani SG. A finite element/control volume approach to mold filling in anisotropic porous media. Polym Compos 1990; 11: 398-405.

[14] Trochu F, Gauvin G, Gao D-M. Numerical analysis of the resin transfer molding process by the finite element method. Adv Polym Technol 1993; 12: 329-342.

[15] Kang MK, Lee WI. A flow-front refinement technique for the numerical simulation of the resin-transfer molding process. Compos Sci Technol 1999; 59: 1663-1674.

[16] Simacek P, Advani SG. A numerical model to predict fiber tow saturation during liquid composite molding. Compos Sci Technol 2003; 63: 1725-1736.

[17] Gourichon B, Binetruy C, Krawczak P. A new numerical procedure to predict dynamic void content in liquid composite molding. Compos Part A 2006; 37: 1961-1969.

[18] Schell JSU, Deleglise M, Binetruy C, Krawczak P, Ermanni P. Numerical prediction and experimental characterisation of meso-scale-voids in liquid composite moulding. Compos Part A 2007; 38: 2460-2470.

[19]Lee DH, Lee WI, Kang MK. Analysis and minimization of void formation during resin 
transfer molding process. Compos Sci Technol 2006; 66: 3281-3289.

[20]Berdichevsky AL, Cai Z. Preform permeability predictions by self-consistent method and finite element simulation. Polym Compos 1993; 14: 132-143.

[21] Xu P, Yu B. Developing a new form of permeability and Kozeny-Carman constant for homogeneous porous media by means of fractal geometry. Adv Water Res 2008; 31: $74-81$.

[22] Liu HL, Hwang WR. Transient filling simulations in unidirectional fibrous porous media. Korea-Australia Rheology Journal 2009; 21:71-79.

[23] Yazdchi K, Srivastava S, Luding S. Micro-macro relations for flow through random arrays of cylinders. Compos Part A 2012; 43:2007-2020.

[24] Soltani P, Johari MS, Zarrebini M. Effect of 3D fiber orientation on permeability of realistic fibrous porous networks. Powder Technol 2014; 254:44-56.

[25] Kundu P, Kumar V, Hoarau Y, Mishra IM. Numerical simulation and analysis of fluid flow hydrodynamics through a structured array of circular cylinders forming porous medium. Appl Math Model 2016; 40:9848-9871.

[26] Bodaghi M, Catalanotti G, Correia N. On the statistics of transverse permeability of randomly distributed fibers. Compos Struct 2016; 158: 323-332.

[27] Okabe T, Matsutani H, Honda T, Yashiro S. Numerical simulation of microscopic flow in 
a fiber bundle using the moving particle semi-implicit method. Compos Part A 2012; 43:

$1765-1774$

[28] Koshizuka S, Nobe A, Oka Y. Numerical analysis of breaking waves using the moving particle semi-implicit method. Int J Numer Methods Fluids 1998; 26: 751-769.

[29]Lu G, He L, Chen D, Li W. Smoothed particle hydrodynamics simulation of dual-scale flow during resin transfer molding. J Reinf Plast Compos 2017; 36: 1431-1438.

[30] Chen Y-T, Davis HT, Macosko CW. Wetting of fiber mats for composites manufacturing: 1. visualization experiments. AICHE J 1995; 41:2261-2273.

[31] Yashiro S. Application of particle simulation methods to composite materials: a review. Adv Compos Mater 2017; 26:1-22.

[32]Röding M, Svensson P, Lorén N. Functional regression-based fluid permeability prediction in monodisperse sphere packings from isotropic two-point correlation functions. Comput Mater Sci 2017; 134:126-131.

[33] Tanaka M, Matsunaga T. Stabilization and smoothing of pressure calculation for the moving particle semi-implicit method. J Comput Phys 2010; 229: 4279-4290. 


\section{Figure captions}

Figure 1 Transparent mold with simulated fiber bundles.

Figure 2 Snapshots of the water flow into a bundle. The dotted line indicates the flow front.

Figure 3 Impregnation process of a bundle with oil. The dotted line indicates the flow front.

Figure 4 Schematic of the estimation of inter-particle potential force.

Figure 5 Final shape of the droplet on a quartz plate.

Figure 6 Analytical model of the transverse flow into a fiber bundle.

Figure 7 Predicted process of impregnation of a bundle with water.

Figure 8 Predicted process of impregnation of a bundle with oil.

Figure 9 Analytical model for predicting the void fraction with a transverse flow.

Figure 10 Analytical results of a transverse flow in fiber arrays. 
Table 1 Material properties of liquids.

\begin{tabular}{llll} 
& Water & Olive oil & Measurement method \\
Density $\left(\mathrm{kg} / \mathrm{m}^{3}\right)$ & $1000\left(^{*}\right)$ & 914 & Archimedes method \\
Kinematic viscosity $\left(\mathrm{m}^{2} / \mathrm{s}\right)$ & $1.0 \times 10^{-6}(*)$ & $8.37 \times 10^{-5}$ & Capillary viscometer method \\
Surface tension $(\mathrm{mN} / \mathrm{m})$ & $72.8(*)$ & 34.6 & Pendant drop method \\
Contact angle to quartz $\left({ }^{\circ}\right)$ & 39.9 & 27.4 & A half-angle method \\
\hline
\end{tabular}

(*) Values from literature

Table 2 Parameters of the inter-particle potential model.

\begin{tabular}{lcc}
\hline & Water & Oil \\
\hline & 20 & 200 \\
& & \\
& 0.73 & \\
Effective radius & & \\
\hline
\end{tabular}




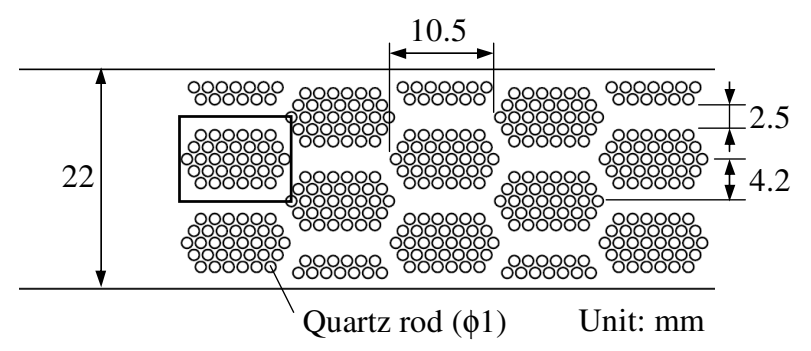

(a) Schematic

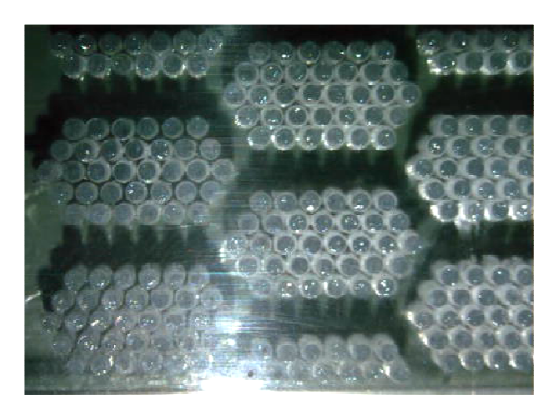

(b) Overview 


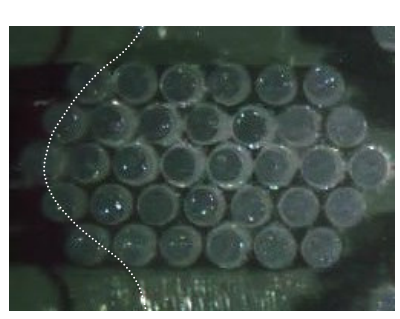

(a) $0.30 \mathrm{~s}$

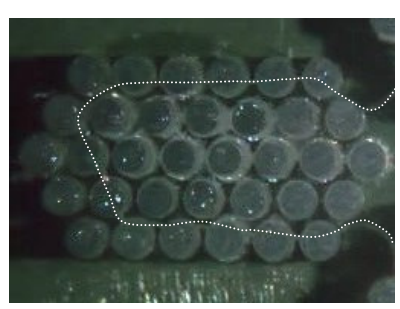

(c) $1.23 \mathrm{~s}$

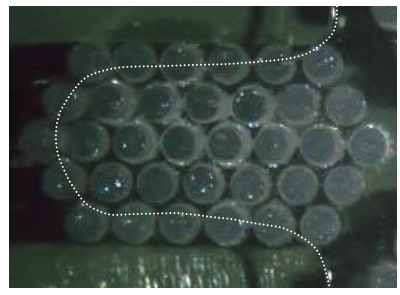

(b) $0.83 \mathrm{~s}$

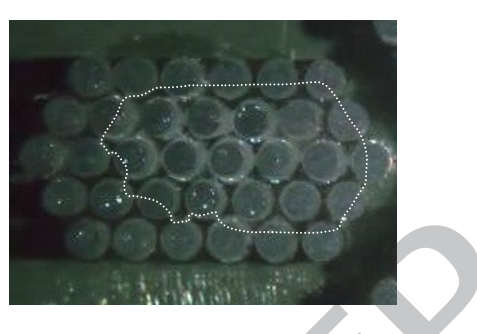

(d) $1.57 \mathrm{~s}$ 


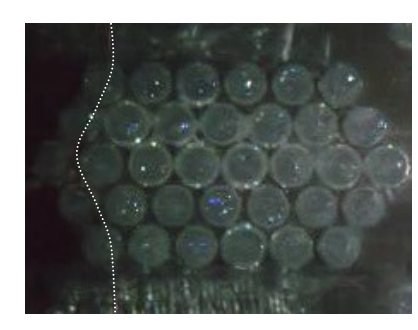

(a) $0.07 \mathrm{~s}$

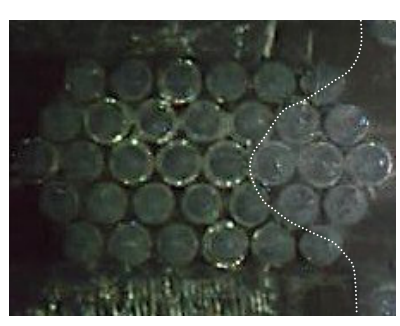

(c) $1.20 \mathrm{~s}$

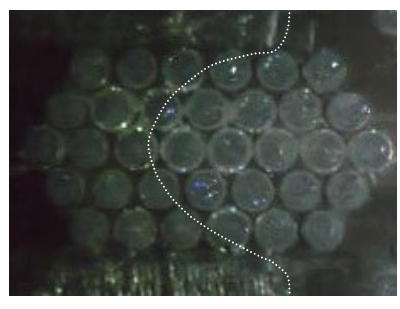

(b) $0.60 \mathrm{~s}$

Fig. 3

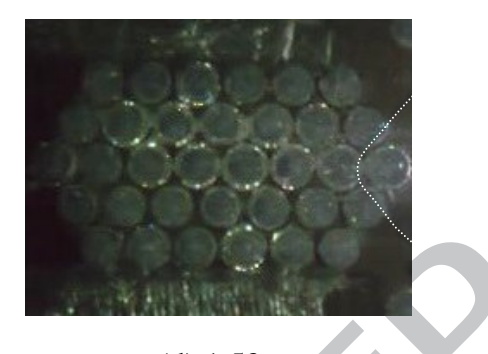

(d) $1.53 \mathrm{~s}$ 


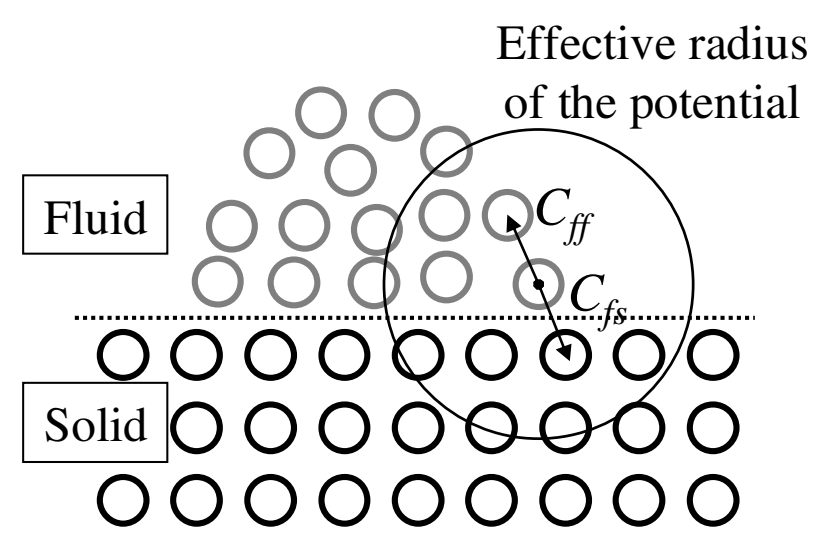




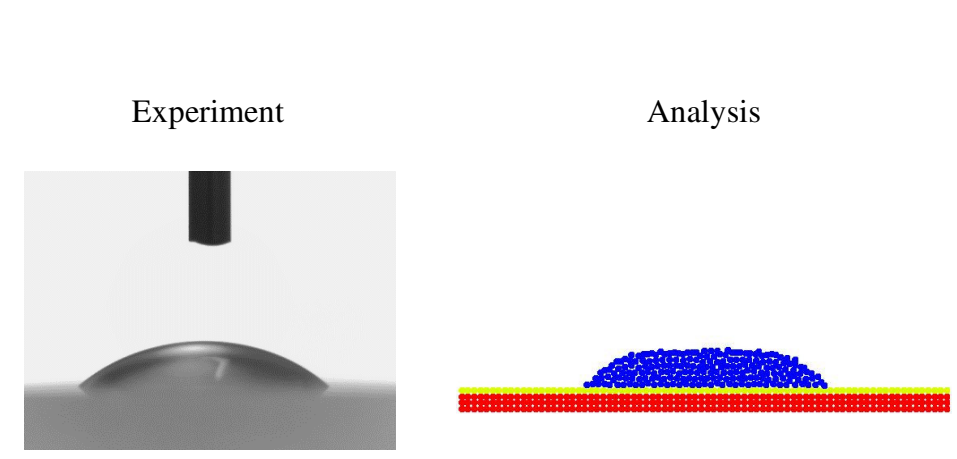

(a) Water

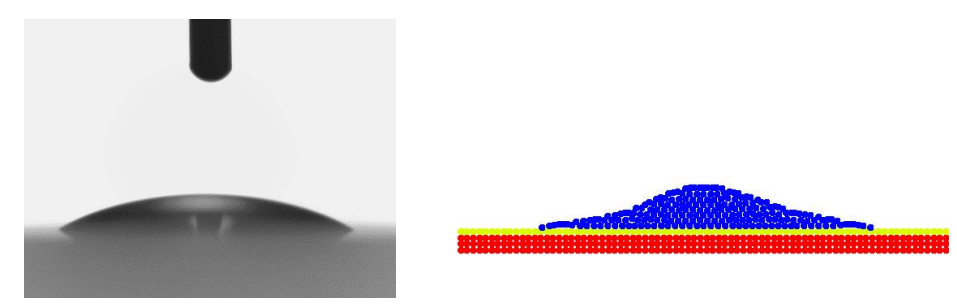

(b) Oil

Fig. 5
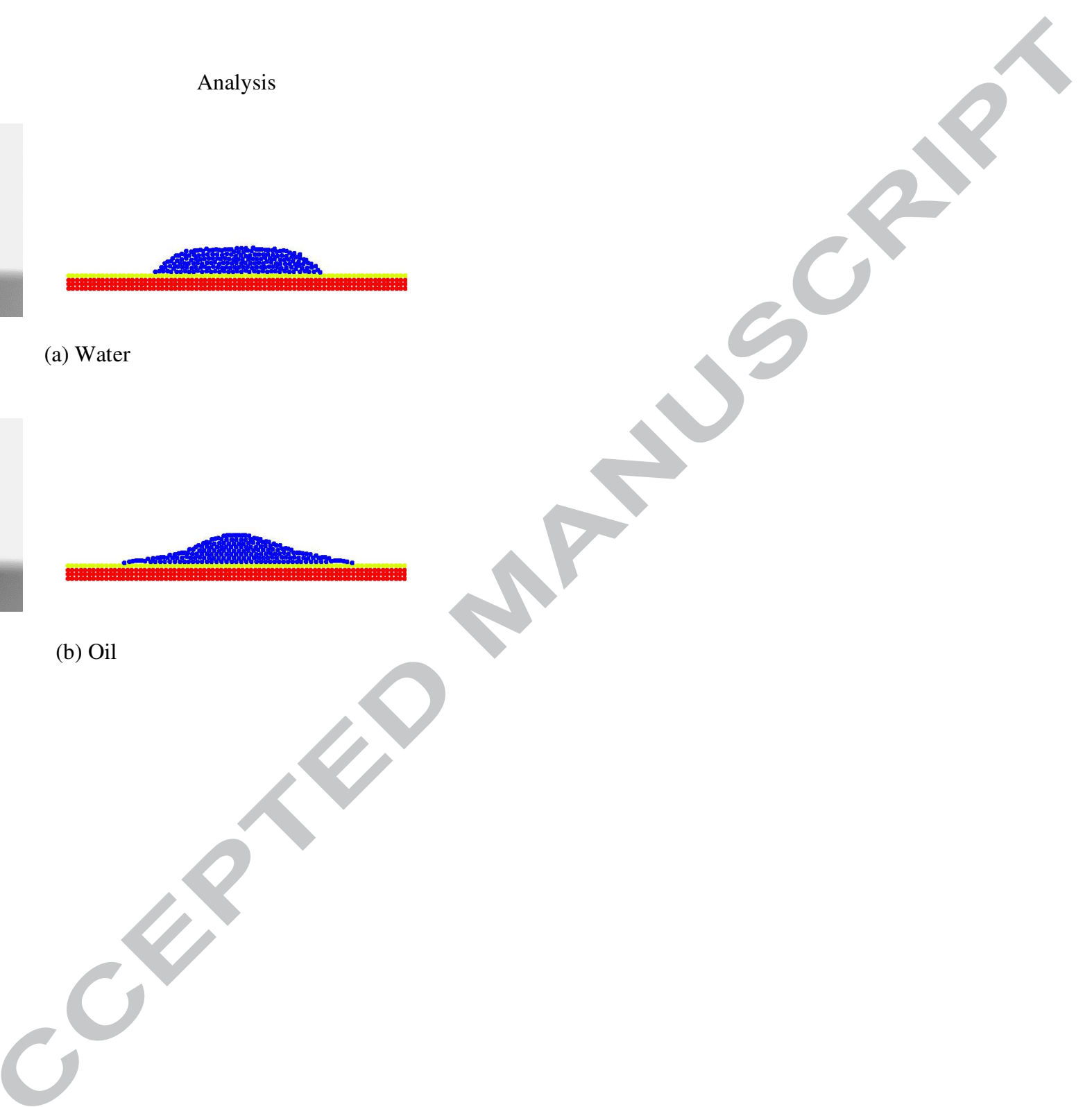


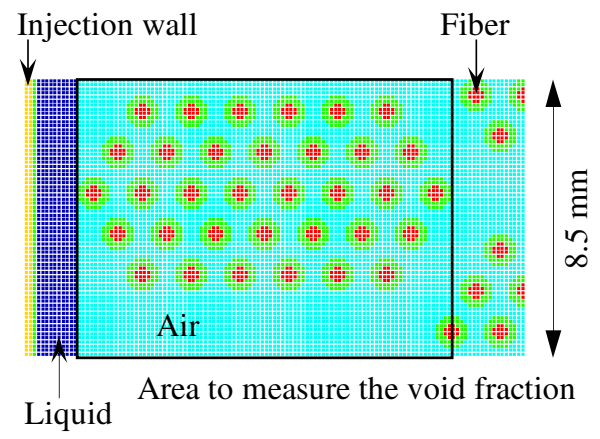

Fig. 6 


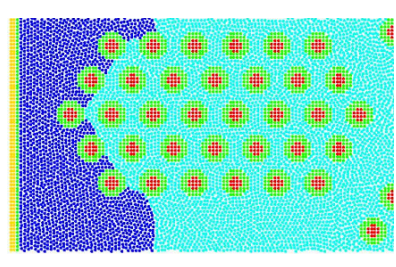

(a) $0.30 \mathrm{~s}$

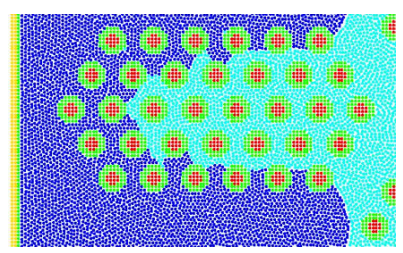

(b) $0.83 \mathrm{~s}$

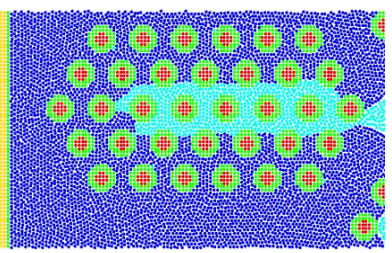

(c) $1.23 \mathrm{~s}$

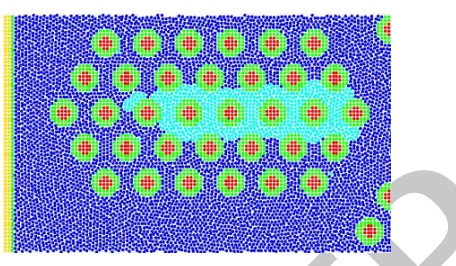

(d) $1.57 \mathrm{~s}$ 


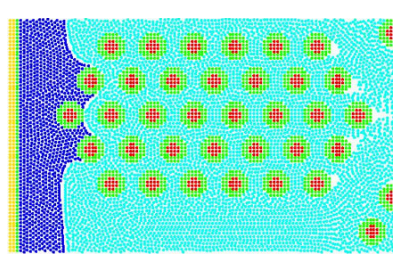

(a) $0.07 \mathrm{~s}$

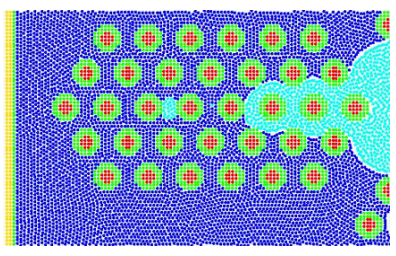

(c) $1.20 \mathrm{~s}$

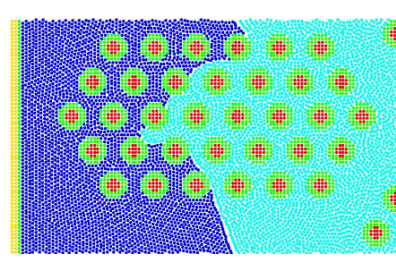

(b) $0.60 \mathrm{~s}$

Fig. 8

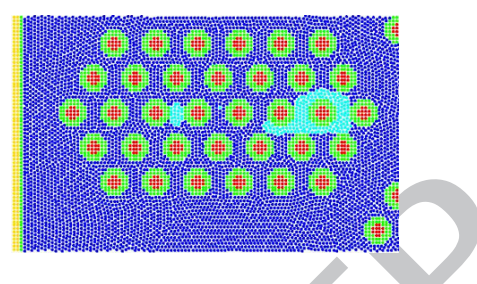

(d) $1.53 \mathrm{~s}$ 


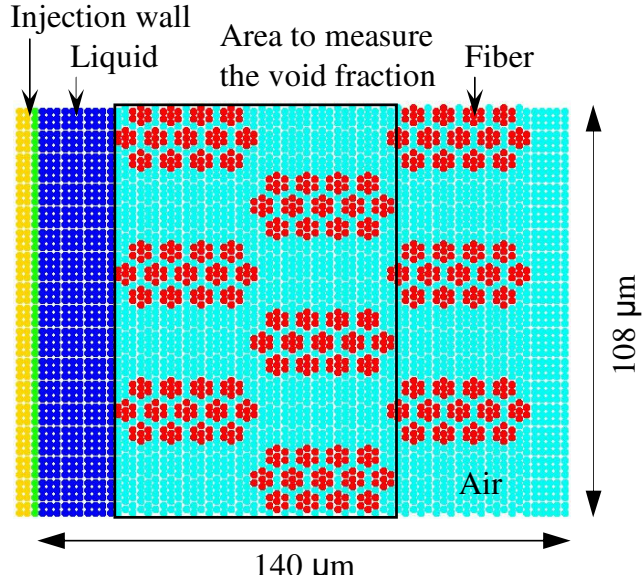




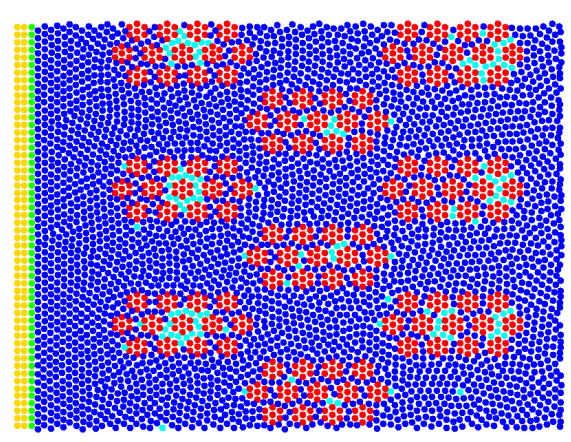

(a) Final particle distribution $\left(\mathrm{Ca}^{*}=7.9\right)$

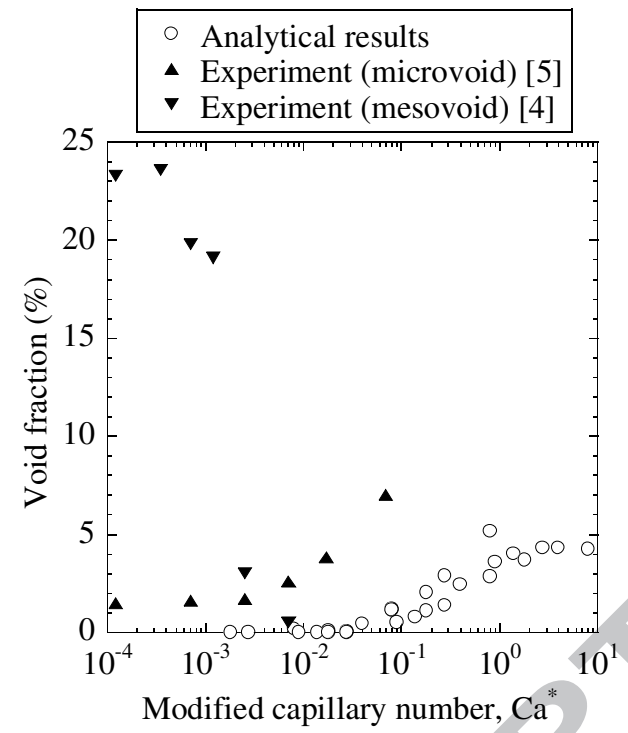

(b) Void fraction as a function of the modified capillary number 